\title{
Constrained normalization of Hamiltonian systems and perturbed Keplerian motion
}

By Jan-Cees van der Meer, Center for Mathematics and Computer Science (CWI), Kruislaan 413, 1098 SJ Amsterdam, and Richard Cushman, Mathematics Institute, Rijksuniversiteit Utrecht, Budapestlaan 6, 3584 CD Utrecht, The Netherlands.

\section{Introduction}

In this paper we develop a theory for normalizing constrained Hamiltonian systems. We make use of some ideas of Moser [6] concerning constrained Hamiltonian systems (see also [2]). The idea of constrained normalization is the following. Consider a Hamiltonian system with Hamiltonian function $H$ on $\left(\mathbb{R}^{2 n}, \omega\right)$, where $\omega$ is the standard symplectic form. Denote such a system by $\left(H, \mathbb{R}^{2 n}, \omega\right)$. For a symplectic submanifold $M \subset \mathbb{R}^{2 n}$ define the constrained system corresponding to $\left(H, \mathbb{R}^{2 n}, \omega\right)$ by $(H|M, M, \omega| M)$. Here $\mid M$ means restriction to $M$. We give a normalization algorithm for the system $\left(H, \mathbb{R}^{2 n}, \omega\right)$ which on $M$ restricts to a normalization of the constrained system. The advantage is that the necessary computations are performed in the ambient space $\mathbb{R}^{2 n}$, where they are easier to do.

The paper is organized as follows. In the second section we give the facts about constrained Hamiltonian systems needed for the development of the constrained normalization algorithm in section three. In the fourth section we introduce the Kepler system on $\mathbb{R}^{2 n}$. As is well known (see [5]) the Kepler system, after regularization, can be considered as a system on $\mathbb{R}^{2 n+2}$ constrained to $T^{+} S^{n}$, the cotangent bundle to the $\mathrm{n}$-sphere minus its zero section. The same techniques enable us to consider perturbed Kepler systems as constrained systems, as is shown in section five. The facts proved in section four show that we may apply the constrained normalization algorithm to perturbed Keplerian systems. We illustrate this with two examples: (i) the lunar problem (section six), and (ii) the main problem of artificial satellite theory (section seven). The treatment of the main problem takes as its starting point the results of Deprit [3] concerning the elimination of the parallax. The normalization up to second order of the lunar problem provides a straightforward alternative for the quite different approach of Kummer [4]. 


\section{Constrained Hamiltonian systems}

Consider $\mathbb{R}^{2 n}$ with coordinates $\left(x_{1}, \ldots, x_{n}, y_{1}, \ldots, y_{n}\right)$ and standard symplectic form $\omega(x, y)=\sum_{i=1}^{n} \mathrm{~d} x_{i} \wedge \mathrm{d} y_{i}$. For $m<n$ let $F_{1}, \ldots, F_{2 m} \in C^{\infty}\left(\mathbb{R}^{2 n}\right)$ be such that $\mathrm{d} F_{1}, \ldots, \mathrm{d} F_{2 m}$ are independent on $M=\left\{(x, y) \in \mathbb{R}^{2 n} \mid F_{1}(x, y)\right.$ $\left.=F_{2}(x, y)=\cdots=F_{2 m}(x, y)=0\right\}$, that is, $M$ is a smoothly embedded submanifold of $\mathbb{R}^{2 n}$. Furthermore suppose that the matrix $C=\left(c_{i j}\right)=\left(\left\{F_{i}, F_{j}\right\}\right)$ is nonsingular at every point of $M$. Then $M$ is a symplectic manifold with symplectic form $\omega \mid M$, the restriction of the symplectic form $\omega$ to $M$.

For $H \in C^{\infty}\left(\mathbb{R}^{2 n}\right)$ the restriction of the Hamiltonian vector field $X_{H}$ to $M$ need not be tangential to $M$. However we can construct a vector field tangential to $M$ by considering $X_{H \mid M}$ on $(M, \omega \mid M)$, where $H \mid M$ is the restriction of $H$ to $M$. We call $X_{H \mid M}$ the constrained Hamiltonian vector field corresponding to $H$. Another way to describe the constrained vector field is that $X_{H \mid M}$ is the image of the projection of $X_{H}$ on $T M$ with respect to the splitting of $T \mathbb{R}^{2 n}$ into $T M$ and its $\omega$-orthogonal complement.

Let $\mathscr{I}$ be the ideal of $C^{\infty}\left(\mathbb{R}^{2 n}\right)$ generated by $F_{1}, \ldots, F_{2 m}$, that is, $\mathscr{I}$ is the ideal of functions vanishing on $M$. Furthermore let $L_{H}$ denote the derivative defined by $L_{H}=\{., H\}$, where $\{.,$.$\} is the Poisson bracket on \mathbb{R}^{2 n}$ with respect to the symplectic form $\omega$.

Lemma 1. The following statements are equivalent:

(i) $X_{H \mid M}=X_{H}$ on $M$.

(ii) $\left\{H, F_{j}\right\} \in \mathscr{I}$, for $j=1, \ldots, 2 m$.

(iii) $\left(\exp L_{H}\right)(\mathscr{I}) \subseteq \mathscr{I}$.

(iv) $M$ is an invariant manifold of $X_{H}$.

(v) $X_{H}$ is tangent to $M$ at each point of $M$.

Proof. The proof is easy and left to the reader.

Let $H \in C^{\infty}\left(\mathbb{R}^{2 n}\right)$. When $X_{H}$ is not tangent to $M$ we can construct a function $\mathbf{H}$ such that $\mathbf{H}|M=H| M, X_{\mathbf{H}}$ is tangent to $M$, and $X_{\mathbf{H}} \mid M=X_{H \mid M}$. The construction of $\mathbf{H}$ is given in Lemma 2. Note that $\mathbf{H}$ need not be a smooth function on all of $\mathbb{R}^{2 n}$. In fact $\mathbf{H}$ is first constructed on $M$ and then extended to some open neighborhood of $M$ in $\mathbb{R}^{2 n}$. Let $C^{-1}=\left(c^{i j}\right)$ be the inverse of the matrix $C$. on $M$.

Lemma 2. If $\mathbf{H}=H+\sum_{i=1}^{2 m} \alpha_{i} F_{i}$, with $\alpha_{i}=\sum_{j=1}^{2 m} c^{i j}\left\{H, F_{j}\right\}$, then $X_{H \mid M}=X_{\mathbf{H}}$ 
Proof. In order for $X_{\mathbf{H}}$ to be tangential to $M$ we must have $0=\left\{\mathbf{H}, F_{j}\right\}$ $=\left\{H, F_{j}\right\}-\sum_{i=1}^{2 m} \alpha_{i}\left\{F_{j}, F_{i}\right\}$ on $M$ for $j=1, \ldots, 2 m$. This holds for $\alpha_{i}$ as given in the statement of the lemma. By Lemma 1 we have $X_{\mathbf{H}}=X_{\mathbf{H} \mid M}$. Because $\mathbf{H}|M=H| M$ we have $X_{\mathbf{H}}=X_{H \mid M}$ on $M$.

The Poisson bracket $\{., .\}^{M}$ on $(M, \omega \mid M)$ can be computed in terms of the Poisson bracket on $\mathbb{R}^{2 n}$ by the following

Lemma 3. $\{H|M, G| M\}^{M}=\{H, G\}-\sum_{i, j=1}^{2 m}\left\{H, F_{i}\right\} c^{i j}\left\{F_{j}, G\right\}$ on $M$, where the right hand side is calculated for any smooth extension of $H \mid M$ and $G \mid M$ to an open neighborhood of $M$ in $\mathbb{R}^{2 n}$.

Proof. (see [2]). $\{H|M, G| M\}^{M}=(\omega \mid M)\left(X_{H \mid M}, X_{G \mid M}\right)$ $=(\omega \mid M)\left(X_{\mathbf{H} \mid M}, X_{\mathbf{G} \mid M}\right)=\omega\left(X_{\mathbf{H}}, X_{\mathbf{G}}\right)=\{\mathbf{H}, \mathbf{G}\}$ on $M$. Computing $\{\mathbf{H}, \mathbf{G}\}$, omitting terms in $\mathscr{I}$, proves the lemma.

As a direct consequence of Lemma 3 we have,

Lemma 4. If $X_{H \mid M}=X_{H}$ on $M$ then $\{H|M, G| M\}^{M}=\{H, G\}$ on $M$ for all $G \in C^{\infty}\left(\mathbb{R}^{2 n}\right)$.

Proof. If $X_{H \mid M}=X_{H}$ then $\left\{H, F_{i}\right\} \in \mathscr{I}$ for all $i=1, \ldots, 2 \mathrm{~m}$. Consequently $\sum_{i, j=1}^{2 m}\left\{H, F_{i}\right\} c^{i j}\left\{F_{j}, G\right\}$ vanishes on $M$. In other words $\{H|M, G| M\}^{M}$ $=\{H, G\} \mid M$.

\section{Constrained normalization}

Consider a Hamiltonian system on $\mathbb{R}^{2 n}$ with Hamiltonian function

$H^{\varepsilon}: \mathbb{R}^{2 n} \rightarrow \mathbb{R} ;(x, y) \rightarrow H_{0}(x, y)+\varepsilon \tilde{H}(x, y, \varepsilon)$

which satisfies the following conditions:

(C1) $H_{0} \in C^{\infty}\left(\mathbb{R}^{2 n}\right)$ and $X_{H_{0}}$ has only periodic orbits.

(C2) The flow of $X_{H_{0}}$ leaves invariant a symplectic manifold $M \cong \mathbb{R}^{2 n}$, where $M$ is defined as in $\S 2$.

(C3) $\tilde{H} \in \mathscr{F}$ where $\mathscr{F}$ is the algebra of formal power series in $\varepsilon$ with coefficients in $C^{\infty}\left(\mathbb{R}^{2 n}\right)$.

Following Cushman [1] we can transform $H^{\varepsilon}$ into normal form with respect to $H_{0}$ by invertible $\omega$-symplectic formal power series transformations. That is, 
there exists a transformation of the form $\exp L_{R}, R \in \mathscr{F}$, such that for $\mathscr{H}^{z}=H^{\varepsilon} \circ \exp L_{R}$ we have $\left\{\mathscr{H}_{m}, H_{0}\right\}=0$ for every $m \in \mathbb{N}$, where $\mathscr{H}_{m}$ is the coefficient of $\varepsilon^{m}$ in $\mathscr{H}^{\varepsilon}$. We say that $\mathscr{H}^{\varepsilon}$ is a normal form for $H^{\varepsilon}$ with respect to $H_{0}$. As a consequence of Lemma 4 we have,

Theorem 5. If $H^{\varepsilon}$ is in normal form up to order $k$ with respect to $H_{0}$, then $H^{\varepsilon} \mid M$ is in normal form up to order $k$ with respect to $H_{0} \mid M$.

Proof. If $H^{\varepsilon}=H_{0}+\varepsilon H_{1}+\varepsilon^{2} H_{2}+\cdots$ is in normal form up to order $k$ then $\left\{H_{0}, H_{l}\right\}=0$ for $0<l \leqq k$. Because $H^{\varepsilon}\left|M=H_{0}\right| M+\varepsilon H_{1} \mid M+\cdots$ by Lemma $4\left\{H_{0}\left|M, H_{l}\right| M\right\}^{M}=0$ for $0<l \leqq k$ on $M$.

A normal form for $H^{\varepsilon}$ is obtained by transformations of the form $\exp L_{R}, R \in \mathscr{F}$. In general these transformations do not restrict to transformations of $M$ into itself. We will show that one can modify the transformations $\exp L_{R}$ in such a way that they restrict to transformations of $M$ into $M$, and such that the restriction of the transformed power series to $M$ gives a normal form for $H^{\varepsilon} \mid M$. This procedure is called constrained normalization or normalization modulo $\mathscr{I}$. Note that, because we will make use of the construction of Lemma 2 , the procedure of constrained normalization is performed on some open neighborhood of $M$ in $\mathbb{R}^{2 n}$.

Definition 6. $H_{0}^{\varepsilon} \exp L_{R}, R \in \mathscr{F}$, is in normal form up to order $k$ with respect to $H_{0}$ modulo the ideal $\mathscr{I}$ if

(N1) $\left\{R, F_{j}\right\} \in \mathscr{F}$ for all $j=1, \ldots, 2 m$.

(N2) All terms in $H^{\varepsilon} \circ \exp L_{R}$ of order $\leqq k$ are in $\left(\operatorname{ker} L_{H_{0}}\right)+\mathscr{I}$.

Here $\mathrm{M}$ and $\mathscr{I}$ are as defined in $\S 2$.

We will now perform the first step in the constrained normalized of $H^{\varepsilon}$. Write $H^{\varepsilon}=H_{0}+\varepsilon H_{1}+O\left(\varepsilon^{2}\right)$. Following [1] we have

$$
C^{\infty}\left(\mathbb{R}^{2 n}\right)=\operatorname{ker} L_{H_{0}} \oplus \operatorname{im} L_{H_{0}}
$$

because $H_{0}$ satisfies (C1). This splitting is obtained by averaging over the flow $\varphi_{t}^{H_{0}}$ of $X_{H_{0}}$. In more detail, for $F \in C^{\infty}\left(\mathbb{R}^{2 n}\right)$ we have $F=\bar{F}+(F-\bar{F})$, where $\bar{F} \in \operatorname{ker} L_{H_{0}}$ is the average of $F$ over the flow of $X_{H_{0}}$, that is,

$$
\bar{F}(p)=\frac{1}{T(p)} \int_{0}^{T(p)}\left(\varphi_{t}^{H_{0}}\right)^{*} F(p) \mathrm{d} t .
$$

Here $T(p)$ is the period of the integral curve of $X_{H_{0}}$ through $p$ and $\left(\varphi_{t}^{H_{0}}\right)^{*} F(p)=F\left(\varphi_{t}^{H_{0}} p\right)$. Thus $H_{1}=\bar{H}_{1}+\hat{H}_{1}$, with $\bar{H}_{1} \in \operatorname{ker} L_{H_{0}}, \quad$ and $\hat{H}_{1}=H_{1}-\bar{H}_{1} \in \operatorname{im} L_{H_{0}}$. Now choose $R_{1} \in C^{\infty}\left(\mathbb{R}^{2 n}\right)$ such that $L_{H_{0}} R_{1}=\hat{H}_{1}$. Then $H^{\varepsilon} \circ \exp L_{\varepsilon R_{1}}=H_{0}+\varepsilon \bar{H}_{1}+\varepsilon \hat{H}_{1}+\varepsilon L_{R_{1}} H_{0}+O\left(\varepsilon^{2}\right)=H_{0}+\varepsilon \bar{H}_{1}+O\left(\varepsilon^{2}\right)$. 
Consequently $H^{\varepsilon} \circ \exp L_{\varepsilon R_{1}}$ is in normal form with respect to $H_{0}$ to first order. The generating function $R_{1}$ for the transformation $\exp L_{\varepsilon R_{1}}$ can be obtained from the following

Lemma 7. [1]. Let $F \in C^{\infty}\left(\mathbb{R}^{2 n}\right)$. If $\bar{F}=0$, then $L_{H_{0}} R=F$ is solved by

$$
R(p)=\frac{1}{T(p)} \int_{0}^{T(p)}\left(t\left(\varphi_{t}^{B_{0}}\right)^{*} F\right)(p) \mathrm{d} t
$$

The above is the usual procedure for normalization of $H^{\varepsilon}$ on $\mathbb{R}^{2 n}$. However, $\exp L_{\varepsilon R_{1}}$ will in general not be a transformation leaving $M$ invariant. Therefore we consider $\exp L_{\varepsilon R_{1}}$ where $\mathbf{R}_{1}$ is defined as in Lemma 2. By Lemma 1, $\exp L_{\varepsilon \mathbf{R}_{1}}$ leaves $M$ invariant. We have

$$
H^{\varepsilon} \circ \exp L_{\varepsilon \mathbf{R}_{1}}=H_{0}+\varepsilon H_{1}+\varepsilon L_{\mathbf{R}_{1}} H_{0}+O\left(\varepsilon^{2}\right),
$$

where $L_{\mathbf{R}_{1}} H_{0}=\left\{H_{0}, R_{1}\right\}+\left\{H_{0}, \sum_{i=1}^{2 m} \alpha_{i} F_{i}\right\}$, with $\alpha_{i}=\sum_{j=1}^{2 m} c^{i j}\left\{R_{1}, F_{j}\right\}$. Write $I=\left\{H_{0}, \sum_{i=1}^{2 m} \alpha_{i} F_{i}\right\}$. Because $\sum_{i=1}^{2 m} \alpha_{i} F_{i} \in \mathscr{I}$, by Lemma $1, I \in \mathscr{I}$ too. Thus $\left\{H_{0}, \mathbf{R}_{1}\right\}$ $=-\hat{H}_{1}+I, I \in \mathscr{I}$. Writing $H_{1}=\left(\bar{H}_{1}+I\right)+\left(\hat{H}_{1}-I\right)$ we have

$$
H^{\varepsilon} \circ \exp L_{e \mathbf{R}_{1}}=H_{0}+\varepsilon\left(\bar{H}_{1}+I\right)+O\left(\varepsilon^{2}\right),
$$

where $\bar{H}_{1}+I \in\left(\operatorname{ker} L_{H_{0}}\right)+\mathscr{I}$. Thus $H^{\varepsilon} \circ \exp L_{\varepsilon \mathbb{R}_{1}}$ is in normal form modulo $\mathscr{I}$ up to order one. By repeating the above argument we can bring $H^{\varepsilon}$ into normal form modulo $\mathscr{I}$ up to arbitrary order.

Theorem 8. Suppose $H^{\varepsilon}$ satisfies conditions (C1), (C2), and (C3), then for each $k \in \mathbb{N}, k>0$, there exists an $R \in \mathscr{F}$ such that $H^{\varepsilon} \circ \exp L_{R}$ is in normal form with respect to $H_{0}$ modulo $\mathscr{I}$.

Remarks. Note that $R$ is defined on some open neighborhood of $M$ in $\mathbb{R}^{2 n}$. The fact that $\omega$ is chosen to be the standard symplectic form on $\mathbb{R}^{2 n}$ is not really necessary. In fact $\omega$ can be any symplectic form. The above normalization procedure still works if one takes the Poisson bracket corresponding to the chosen symplectic form.

In some cases the function $I \in \mathscr{I}$ in the constrained normalization construction takes a special form. This is shown in the following theorem.

Theorem 9. Suppose that the manifold $M$ is defined by $F_{1}(x, y)=F_{2}(x, y)$ $=0$. Furthermore suppose that $\left\{H, F_{i}\right\}=\alpha_{i} F_{1}+\beta_{i} F_{2}, i=1,2$, where $\alpha_{i}$ and $\beta_{i}$ are constants. Then for every $G \in C^{\infty}\left(\mathbb{R}^{2 n}\right)$ we have $\{H, \mathbf{G}\}=\mathbf{E}$, where $E=\{H, G\}$ 
Proof. Using Lemma 2 we have

$$
\begin{aligned}
\{H, \mathbf{G}\}= & \{H, G\}+\frac{\left\{H, F_{1}\right\}\left\{F_{2}, G\right\}}{\left\{F_{1}, F_{2}\right\}}+\frac{\left\{H,\left\{F_{2}, G\right\}\right\} F_{1}}{\left\{F_{1}, F_{2}\right\}} \\
& -\frac{\left\{H,\left\{F_{1}, F_{2}\right\}\right\}\left\{F_{2}, G\right\} F_{1}}{\left\{F_{1}, F_{2}\right\}^{2}}+\frac{\left\{H, F_{2}\right\}\left\{F_{1}, G\right\}}{\left\{F_{2}, F_{1}\right\}} \\
& +\frac{\left\{H,\left\{F_{1}, G\right\}\right\} F_{2}}{\left\{F_{2}, F_{1}\right\}}-\frac{\left\{H,\left\{F_{2}, F_{1}\right\}\right\}\left\{F_{1}, G\right\} F_{2}}{\left\{F_{2}, F_{1}\right\}^{2}} \\
= & \{H, G\}+\frac{1}{\left\{F_{1}, F_{2}\right\}}\left[\left\{H, F_{1}\right\}\left\{F_{2}, G\right\}-\left\{H, F_{2}\right\}\left\{F_{1}, G\right\}\right. \\
& +\left\{\left\{H, F_{2}\right\}, G\right\} F_{1}+\left\{\{G, H\}, F_{2}\right\} F_{1}-\left\{\left\{H, F_{1}\right\}, G\right\} F_{2} \\
& \left.-\left\{\{G, H\}, F_{1}\right\} F_{1}-\left\{\left\{H, F_{1}\right\}, F_{2}\right\}(\mathbf{G}-G)-\left\{\left\{F_{2}, H\right\}, F_{1}\right\}(\mathbf{G}-G)\right]
\end{aligned}
$$

By hypothesis we may write $\left\{H, F_{i}\right\}=\alpha_{i} F_{1}+\beta_{i} F_{2}, i=1,2$, where $\alpha_{i}, \beta_{i}$ are constants. Substitution then gives

$$
\begin{aligned}
\{H, \mathbf{G}\} & =\{H, G\}+\frac{\left\{F_{2},\{H, G\}\right\} F_{1}}{\left\{F_{1}, F_{2}\right\}}+\frac{\left\{F_{1},\{H, G\}\right\} F_{2}}{\left\{F_{2}, F_{1}\right\}} \\
& =E+\frac{\left\{F_{2}, E\right\} F_{1}}{\left\{F_{1}, F_{2}\right\}}+\frac{\left\{F_{1}, E\right\} F_{2}}{\left\{F_{2}, F_{1}\right\}}=\mathbf{E} .
\end{aligned}
$$

If $H_{0}$ and $M$ satisfy the hypothesis of Theorem 9 we may slightly adjust our normalization modulo $\mathscr{I}$ to obtain a somewhat nicer normal form. We again will perform the normalization process up to first order. Instead of $H^{\varepsilon}=H_{0}+\varepsilon H_{1}+O\left(\varepsilon^{2}\right)$ we consider $H_{0}+\varepsilon \mathbf{H}_{1}+O\left(\varepsilon^{2}\right)$. By Lemma 1 (iii) this will not change the restriction to $M$ of the normalized function. Now if $L_{R_{1}} H_{0}=-\hat{H}_{1}$, then by Theorem $9, L_{\mathbf{R}_{1}} H_{0}=-\hat{\mathbf{H}}_{1}$. Because $\mathbf{H}_{1}=\overline{\mathbf{H}}_{1}+\hat{\mathbf{H}}_{1}$ the constrained normal form up to first order is $H_{0}+\varepsilon \mathbf{H}_{1}+O\left(\varepsilon^{2}\right)$. Applying the same procedure up to order $k$ gives a normal form which can be written as $H^{\varepsilon}=H_{0}+\varepsilon \overline{\mathbf{H}}_{1}+\varepsilon^{2} \overline{\mathbf{H}}_{2}+\cdots+\varepsilon^{k} \overline{\mathbf{H}}_{k}+O\left(\varepsilon^{k+1}\right)$, where $\bar{H}_{l} \in \operatorname{ker} L_{H_{0}}$, $0<l \leqq k$.

\section{The Kepler system as a constrained oscillator}

Consider the Kepler system $\left(K_{0}, M, \omega_{2 n} \mid M\right)$, where $M=\left(\mathbb{R}^{n}-\{0\}\right) \times \mathbb{R}^{n}$,

$$
K_{0}(\xi, \eta)=\frac{1}{2}|\eta|^{2}-\frac{\mu}{|\xi|},
$$

and $\omega_{2 n}=\sum_{i=1}^{n} \mathrm{~d} \xi_{i} \wedge \mathrm{d} \eta_{i}$ is the standard symplectic form on $\mathbb{R}^{2 n}$. Here $|$.$| is the$ norm associated to the euclidean inner product $\langle.,$.$\rangle .$ 
In this section we will show how constraining the oscillator system $\left(H_{0}, N, \omega_{2 n+2} \mid N\right)$ to $\left(T^{+} S^{n}, \omega_{2 n+2} \mid T^{+} S^{n}\right)$ gives the Kepler system on the punctured cotangent bundle

$$
T^{+} S^{n}=\left\{\left.(q, p) \in \mathbb{R}^{2 n+2}|| q\right|^{2}=1,\langle q, p\rangle=0, p \neq 0\right\},
$$

of $S^{n}$. Here the Hamiltonian of the oscillator is

$$
H_{0}(q, p)=\left(|q|^{2}|p|^{2}-\langle q, p\rangle^{2}\right)^{1 / 2},
$$

and the phase space is

$$
N=\mathbb{R}^{2 n+2}-C_{2 n+2},
$$

where $C_{2 n+2}=\left\{\left.(q, p) \in \mathbb{R}^{2 n+2}|| q\right|^{2}|p|^{2}=\langle q, p\rangle^{2}\right\}$.

Converting $\left(K_{0}, M, \omega_{2 n} \mid M\right)$ into $\left(H_{0}, N, \omega_{2 n+2} \mid N\right)$ is based upon the regularization given in Moser [5]. The regularization of the Kepler system consists of a pre-regularization followed by a sympletic map. We start with the preregularization.

The pre-regularized Kepler Hamiltonian is given by

$$
\hat{K}_{0}=\frac{|\xi|}{k}\left(K_{0}+\frac{1}{2} k^{2}\right)+\frac{\mu}{k}=\frac{1}{2 k}|\xi|\left(|\eta|^{2}+k^{2}\right) \text {. }
$$

On the energy surface $\hat{K}_{0}=\frac{\mu}{k}=L$ (which corresponds to the level set $K_{0}=-\frac{1}{2} k^{2}$ ) the Hamiltonian vector field of the pre-regularized Kepler Hamiltonian $\hat{K}_{0}$ is given by

$$
\begin{aligned}
& \frac{\mathrm{d} \xi}{\mathrm{d} s}=\left.\frac{\partial \hat{K}_{0}}{\partial \eta}\right|_{K_{0}=-\frac{1}{2} k^{2}}=\left.\left[\frac{1}{k}|\xi| \frac{\partial K_{0}}{\partial \eta}+\left(K_{0}+\frac{1}{2} k^{2}\right) \frac{\partial}{\partial \eta} \frac{|\xi|}{k}\right]\right|_{K_{0}=-\frac{1}{2} k^{2}}=\frac{1}{k}|\xi| \frac{\partial K_{0}}{\partial \eta} \\
& \frac{\mathrm{d} \eta}{\mathrm{d} s}=-\left.\frac{\partial \hat{K}_{0}}{\partial \xi}\right|_{K_{0}=-\frac{1}{2} k^{2}}=-\frac{1}{k}|\xi| \frac{\partial K_{0}}{\partial \xi}
\end{aligned}
$$

In other words on $\hat{K}_{0}=L, X_{\hat{R}_{0}}$ is just the Kepler vector field $X_{K_{0}}$

$$
\begin{aligned}
& \frac{\mathrm{d} \xi}{\mathrm{d} t}=\frac{\partial K_{0}}{\partial \eta}=\eta, \\
& \frac{\mathrm{d} \eta}{\mathrm{d} t}=-\frac{\partial K_{0}}{\partial \xi}=-\frac{\mu \xi}{|\xi|^{3}}
\end{aligned}
$$

in a new time scale $s$ given by $\frac{\mathrm{d} s}{\mathrm{~d} t}=\frac{k}{|\xi|}$. 
Let $T^{+} S_{n p}^{n}=\left\{(q, p) \in T^{+} S^{n} \mid q \neq(0, \ldots, 0,1)\right\}$. Following Moser [5] the system $\left(\hat{K}_{0}, M, \omega_{2 n} \mid M\right)$ is symplectically diffeomorphic to the system $\left(\hat{G}_{0}, T^{+} S_{n p}^{n}, \omega_{2 n+2} \mid T^{+} S_{n p}^{n}\right)$, where

$$
\hat{G}_{0}(q, p)=|p| \text {. }
$$

The desired diffeomorphism $m: T^{+} S_{n p}^{n} \rightarrow M$ is given by

$$
\xi_{i}=-\frac{1}{k}\left(p_{i}\left(1-q_{n+1}\right)+q_{i} p_{n+1}\right), \quad \eta_{i}=\frac{k q_{i}}{1-q_{n+1}}, \quad i=1, \ldots, n .
$$

We call $m$ Moser's regularization map. The inverse map $m^{-1}: M \rightarrow T^{+} S_{n p}^{n}$ is given by

$$
\begin{aligned}
& q_{i}=\frac{2}{k}\left(\frac{1}{k^{2}}|\eta|^{2}+1\right)^{-1} \eta_{i}, \quad q_{n+1}=\left(\frac{1}{k^{2}}|\eta|^{2}+1\right)^{-1}\left(\frac{1}{k^{2}}|\eta|^{2}-1\right), \\
& p_{i}=-\frac{2}{k}\left(\frac{1}{k^{2}}|\eta|^{2}+1\right) \xi_{i}+\frac{1}{k}\langle\eta, \xi\rangle \eta_{i}, \quad p_{n+1}=-\langle\eta, \xi\rangle, \quad i=1, \ldots, n .
\end{aligned}
$$

Since $\hat{G}_{0}$ does not depend on $q$ it extends to a smooth function $G_{0}$ on $\left(T^{+} S^{n}, \omega_{2 n+2} \mid T^{+} S^{n}\right)$, which is the Hamiltonian for the geodesic vector field on $T^{+} S^{n}$. Note that the set $B=T^{+} S^{n}-T^{+} S_{n p}^{n}$ $=\left\{\left(0, \ldots, 0,1, p_{1}, \ldots, p_{n}\right) \in \mathbb{R}^{2 n+2} \mid \tilde{p}=\left(p_{1}, \ldots, p_{n}\right) \neq 0\right\}$ corresponds to collisions in the Kepler system. $B$ is called the collision set. The system $\left(G_{0}, T^{+} S^{n}, \omega_{2 n+2} \mid T^{+} S^{n}\right)$ is called the regularized Kepler system. In the regularized Kepler system a collision orbit can be treated like any other orbit.

Next we show that the system $\left(G_{0}, T^{+} S^{n}, \omega_{2 n+2} \mid T^{+} S^{n}\right)$ can be considered as a constrained oscillator. On the symplectic manifold $\left(N, \omega_{2 n+2} \mid N\right)$, where $N$ is given by (6), consider the Hamiltonian $H_{0}(q, p)$ given by (5). Since

$$
|q|^{2}|p|^{2}-\langle q, p\rangle^{2}=\sum_{1 \leqq i<j \leqq n+1}\left(q_{i} p_{j}-q_{j} p_{i}\right)^{2},
$$

$H_{0}$ is defined and is a smooth positive function on $N$. Since $C_{2 n+2} \cap T^{+} S^{n}=\emptyset$, $H_{0}$ restricted to $T^{+} S^{n}$ is defined and $H_{0} \mid T^{+} S^{n}=G_{0}$.

Lemma 10. $\left(T^{+} S^{n}, \omega_{2 n+2} \mid T^{+} S^{n}\right)$ is a symplectic submanifold of $\left(N, \omega_{2 n+2} \mid N\right)$.

Proof. Let

$$
F_{1}(q, p)=|q|^{2}-1, \quad F_{2}(q, p)=\langle q, p\rangle \text {. }
$$

The matrix $C=\left(\left\{F_{i}, F_{j}\right\}\right), i, j=1,2$, is nonsingular on $N$ because $\left\{F_{1}, F_{2}\right\}(q, p)$ $=2\left(F_{1}(q, p)+1\right)=2|q|^{2}>0$.

Consequently $\left(G_{0}, T^{+} S^{n}, \omega_{2 n+2} \mid T^{+} S^{n}\right)$ is the constrained system on $T^{+} S^{n}$ corresponding to $\left(H_{0}, N, \omega_{2 n+2} \mid N\right)$. 
Lemma 11. $T^{+} S^{n}$ is an invariant manifold for $X_{H_{0}}$.

Proof. As is easily checked $\left\{H_{0}, F_{1}\right\}=0$ and $\left\{H_{0}, F_{2}\right\}=0$, which, using Lemma 1, completes the proof.

We can write down the Hamiltonian vector field on $\left(N, \omega_{2 n+2} \mid N\right)$ corresponding to $H_{0}(q, p)$. Explicitly, we have

$$
X_{H_{0}}(q, p)=\frac{2}{H_{0}(q, p)}\left(\begin{array}{cc}
-\langle q, p\rangle I_{n+1} & |q|^{2} I_{n+1} \\
-|p|^{2} I_{n+1} & \langle q, p\rangle I_{n+1}
\end{array}\right)\left(\begin{array}{l}
q \\
p
\end{array}\right)=A(q, p)\left(\begin{array}{l}
q \\
p
\end{array}\right)
$$

where $I_{n+1}$ is the $(n+1) \times(n+1)$ identity matrix. From the proof of Lemma 11 it follows that $|q|^{2}$ and $\langle q, p\rangle$ are integrals of $X_{H_{0}}$. Since $H_{0}^{2}$ is also an integral, $|p|^{2}$ is an integral of $X_{H_{0}}$. Consequently the matrix $A(q, p)$ is constant along the orbits of $X_{H_{0}}$, that is, the flow of $X_{H_{0}}$ is a linear flow. For convenience let $H_{0}(q, p)=h,|q|^{2}=a,|p|^{2}=b,\langle q, p\rangle=d$, where $h^{2}=a b-d^{2}>0$, then the flow $\varphi_{t}^{H_{0}}$ of $X_{H_{0}}$ is given by the matrix

$$
(\cos 2 t) I_{2 n+2}+\frac{1}{2}(\sin 2 t) A
$$

which is equal to

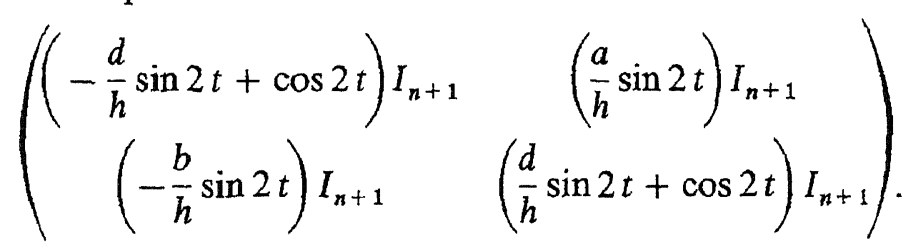

Consequently,

Lemma 12. On $\left(N, \omega_{2 n+2} \mid N\right)$ the flow of $X_{H_{0}}$ is periodic, all integral curves having period $\pi$.

Recall that the vector space $C^{\infty}\left(\mathbb{R}^{2 n+2}\right)$ of smooth functions on $\left(\mathbb{R}^{2 n+2}, \omega_{2 n+2}\right)$ is a Lie algebra under Poisson bracket. A straight forward calculation shows that the smooth functions $\frac{1}{2}|q|^{2}, \frac{1}{2}|p|^{2},\langle q, p\rangle$ span a Lie subalgebra $\mathscr{L}$ of $\left(C^{\infty}\left(\mathbb{R}^{2 n+2}\right),\{. .\}.\right)$, which is isomorphic to $\operatorname{sl}_{2}(\mathbb{R})$. As is easily checked, every smooth function of the quadratic polynomials

$$
S_{i j}=q_{i} p_{j}-q_{i} p_{j}, \quad 1 \leqq i<j \leqq n+1,
$$

lies in the centralizer of $\mathscr{L}$. Consequently every smooth function in the quadratic polynomials $S_{i j}$ commutes with every smooth function on $\mathscr{L}$. Thus we have proved

Lemma 13. $S_{i j} \mid N, 1 \leqq i<j \leqq n+1$, are integrals of $X_{B_{0}}$. 
By Lemma 4 and Lemma 10 it follows that the $S_{i j} \mid T^{+} S^{n}$ are integrals of $X_{G_{0}}$ on $T^{+} S^{n}$. In fact the $S_{i j} \mid T^{+} S^{n}$ are the components of an $S O(n+1, \mathbb{R})$ momentum mapping arising from the linear action of $S O(n+1, \mathbb{R})$ on $\mathbb{R}^{n+1}$ restricted to $S^{n}$ (see [1]). Using (12) a short calculation shows that

$$
J_{i j}=\left(S_{i j} \mid T^{+} S^{n}\right) \circ m^{-1}=\xi_{i} \eta_{j}-\xi_{j} \eta_{i}, \quad 1 \leqq i<j \leqq n,
$$

correspond to the components of an $S O(n, \mathbb{R})$ momentum mapping for the Kepler Hamiltonian $K_{0}$. Since $K_{0}$ is invariant under the $S O(n, \mathbb{R})$-action, the $J_{i j}$ correspond to the integrals of $K_{K_{0}}$. The functions

$$
A_{i}=\left(S_{i n+1} \mid T^{+} S^{n}\right) \circ m^{-1}, \quad i=1, \ldots, n,
$$

on the $L$-level set of $H_{0}$ correspond to the components of the Laplace vector, which also are integrals for $X_{K_{0}}$.

Finally we will determine the orbits of the regularized Kepler system $\left(G_{0}, T^{+} S^{n}, \omega_{2 n+2} \mid T^{+} S^{n}\right)$ which correspond to collision orbits of the Kepler system $\left(K_{0}, M, \omega_{2 n} \mid M\right)$. These are the orbits which pass through the collision set $B=\left\{\left(0, \ldots, 0,1, p_{1}, \ldots, p_{n}, 0\right) \in \mathbb{R}^{2 n+2} \mid\left(p_{1}, \ldots, p_{n}\right) \neq 0\right\}$. Let $\tilde{q}=\left(q_{1}, \ldots, q_{n}\right)$ and $\tilde{p}=\left(p_{1}, \ldots, p_{n}\right)$ be the $n$-vectors consisting of the first $n$ components of the vectors $q$ and $p$ respectively.

Define $\tilde{G}(q, p)=|\tilde{q}|^{2}|\tilde{p}|^{2}-\langle\tilde{q}, \tilde{p}\rangle^{2}$ and $C_{2 n}=\left\{(q, p) \in \mathbb{R}^{2 n+2} \mid \tilde{G}(q, p)=0\right\}$. Because $\widetilde{G}(q, p)=\sum_{1 \leqq i<j \leqq n}\left(q_{i} p_{j}-q_{j} p_{i}\right)^{2}, \widetilde{G} \mid T^{+} S^{n}$ is an integral of $X_{G_{0}}$. Consequently $C_{2 n} \cap T^{+} S^{n}$ is a union of orbits of $X_{G_{0}}$.

Lemma 14. $C_{2 n} \cap T^{+} S^{n}$ is the set of all integral curves of $X_{G_{0}}$ passing through $B$.

Proof. We have to show that $\left\{\varphi_{t}^{H_{0}}(B), 0 \leqq t<\pi\right\}=C_{2 n} \cap T^{+} S^{n}$. Let $\tilde{w}$ denote the $n$-vector obtained by taking the first $n$ components of $w \in \mathbb{R}^{n+1}$. Consider the point $(\tilde{0}, 1, \tilde{p}, 0) \in B$. Then $\varphi_{t}^{H_{0}}(\tilde{0}, 1, \tilde{p}, 0)=\left(\frac{p_{1}}{|\tilde{p}|} \sin 2 t, \ldots\right.$, $\left.\frac{p_{n}}{|\tilde{p}|} \sin 2 t, \cos 2 t, p_{1} \cos 2 t, \ldots, p_{n} \cos 2 t,-|\tilde{p}| \sin 2 t\right)$. It is now easy to check that $\varphi_{t}^{H_{0}}(0,1, \tilde{p}, 0) \in C_{2 n} \cap T^{+} S^{n}$.

Finally we will show that each point in $C_{2 n} \cap T^{+} S^{n}$ is the image of $\varphi_{t}^{H_{0}}$ of some point in $B$. Note that $\tilde{G}(q, p)=0$ is equivalent to the least one of the following three conditions: (i) $\tilde{p}=0$, (ii) $\tilde{q}=0$, (iii) $\tilde{q}=\lambda \tilde{p}, \lambda \in \mathbb{R}, \lambda \neq 0$.

(i) Suppose $(u, v) \in C_{2 n} \cap T^{+} S^{n}, \tilde{u}=0$. From $|u|^{2}=|\tilde{u}|^{2}+u_{n+1}^{2}=1$ we obtain $u_{n+1}= \pm 1$, and from $\langle u, v\rangle=0$ we obtain $v_{n+1}=0$. Consequently $(u, v)=(\tilde{0}, \pm 1, \tilde{v}, 0)$. We have $(\tilde{0}, 1, \tilde{v}, 0) \in B$ and $(\tilde{0},-1, \tilde{v}, 0)=\varphi_{\pi}^{H_{0}}(\tilde{0}, 1, \tilde{v}, 0)$.

(ii) Suppose $(u, v) \in C_{2 n} \cap T^{+} S^{n}, \tilde{v}=0$ Because $(u, v) \in T^{+} S^{n}$ we must have $v_{n+1} \neq 0$. Consequently $u_{n+1}=0$ because of $\langle u, v\rangle=0$. Thus we have 
$(u, v)=\left(\tilde{u}, 0, \widetilde{0}, v_{n+1}\right)$. If $v_{n+1}>0$ then $\left(\tilde{u}, 0, \tilde{0}, v_{n+1}\right)=\varphi_{\frac{3}{4} \pi}^{H_{0}}\left(\tilde{0}, 1, u_{1} \sqrt{v_{n+1}}, \ldots\right.$, $\left.u_{n} \sqrt{v_{n+1}}, 0\right)$, and if $v_{n+1}<0$ then $\left(\tilde{u}, 0, \tilde{0}, v_{n+1}\right)=\varphi_{\frac{1}{4} \pi}^{B_{0}}\left(\tilde{0}, 1, u_{1} \sqrt{-v_{n+1}}, \ldots\right.$, $u_{n} \sqrt{-v_{n+1}}, 0$ ).

(iii) Suppose $(u, v) \in C_{2 n} \cap T^{+} S^{n}, \quad \tilde{u}=\lambda \tilde{v}$. From $|u|^{2}=|\tilde{u}|^{2}+u_{n+1}^{2}=$ $\lambda^{2}|\tilde{v}|^{2}+u_{n+1}^{2}=1$ we have $\lambda^{2}=\frac{1-u_{n+1}^{2}}{|\tilde{v}|^{2}}$. If we choose $t_{0}$ such that $u_{n+1}=$ $\cos 2 t_{0}$ (This can always be done because $|u|^{2}=1$. Thus $u_{n+1} \leqq 1$. There are two choices depending on the sign of $\lambda$ ) then $\left(\lambda \tilde{v}, u_{n+1}, \tilde{v}, \frac{1}{\lambda} u_{n+1}\right)=$ $\varphi_{t_{0}}^{H_{0}}\left(\tilde{0}, 1, \frac{\tilde{v}}{u_{n+1}}, 0\right)$

Since $\tilde{G}$ is an integral of $X_{H_{0}}, V=N-C_{2 n}$ with symplectic form $\omega_{2 n+2} \mid V$ is an invariant symplectic manifold for $X_{H_{0}}$. From Lemma 12 it follows that all the integral curves of $X_{B_{0}} \mid V$ are periodic with period $\pi$. Constraining the system $\left(H_{0}, V, \omega_{2 n+2} \mid V\right)$ to $\mathbf{T}^{+} \mathbf{S}^{n}=T^{+} S^{n}-\left(C_{2 n} \cap T^{+} S^{n}\right)$ gives the system $\left(G_{0}, \mathbf{T}^{+} \mathbf{S}^{n}, \omega_{2 n+2} \mid \mathbf{T}^{+} \mathbf{S}^{n}\right)$ whose integral curves, when projected on $S^{n}$, are geodesics which do not pass through the pole $(0, \ldots, 0,1)$.

\section{Normalization of perturbed Kepler systems}

Consider a perturbed Keplerian system on $\left(M=\left(\mathbb{R}^{n}-\{0\}\right) \times \mathbb{R}^{n}, \omega_{2 n} \mid M\right)$ with Hamiltonian given by

$$
K^{\varepsilon}(\xi, \eta)=K_{0}(\xi, \eta)+\varepsilon K_{1}(\xi, \eta, \varepsilon),
$$

where $K_{0}$ is the Kepler Hamiltonian given by (1), and $K_{1} \in \mathscr{F}$, that is, $K_{1}$ is a formal power series in $\varepsilon$ with coefficients which are smooth on $M . K^{\varepsilon}$ is said to be in normal form if $\left\{K_{0}, K_{1}\right\}=0$. In this section we will show how the formal Hamiltonian (20) can be transformed into normal form using the theory of constrained normalization developed in $\S 3$. Towards this end we first have to describe $\left(K^{\varepsilon}, M, \omega_{2 n} \mid M\right)$ as a constrained system. We do this by following the regularization process for the Kepler system of $\S 4$. We start by applying the pre-regularization to $K^{\varepsilon}$. This gives

$$
\hat{K}^{\varepsilon}(\xi, \eta)=\hat{K}_{0}(\xi, \eta)+\varepsilon \hat{K}_{1}(\xi, \eta, \varepsilon),
$$

where $\hat{K}_{0}$ is the function given by (13), and $\hat{K}_{1}=\frac{|\xi|}{k} K_{1}$, with $K_{1}$ as in (20). Next apply Moser's regularization map $m$ to $\hat{K}^{\varepsilon}$ to obtain a system $\left(\hat{G}^{\varepsilon}, T^{+} S_{n p}^{n}\right.$, $\left.\omega_{2 n+2} \mid T^{+} S_{n p}^{n}\right)$ with

$$
\hat{G}^{\varepsilon}(q, p)=\hat{G}_{0}(q, q)+\varepsilon \hat{G}_{1}(q, p, \varepsilon),
$$

where $\hat{G}_{0}$ is given by $(10)$. 
Now we have to distinguish two cases: (i) $\hat{G}^{\varepsilon}$ can be extended to a power series with smooth coefficients on $T^{+} S^{n}$. (ii) $\hat{G}^{\varepsilon}$ can not be extended to such a power series.

We are in case (i) when $K_{1}$ is at most linear in the coordinates $\eta$. This follows easily from the fact that under $m^{-1},|\xi|$ turns into $\frac{1}{k}|p|\left(1-q_{n+1}\right)$ while $\eta$ turns into $\frac{k q}{1-q_{n+1}}$. It is now clear that under this hypothesis $\hat{G}_{1}$ can be extended to a smooth function on all of $T^{+} S^{n}$. Extending $\hat{G}_{0}$ to $T^{+} S^{n}$ gives us the system on $\left(T^{+} S^{n}, \omega_{2 n+2} \mid T^{+} S^{n}\right)$ with Hamiltonian

$$
G^{\varepsilon}(q, p)=G_{0}(q, p)+\varepsilon \hat{G}_{1}(q, p, \varepsilon)
$$

Because $(q, p)$ are in fact coordinates on $\mathbb{R}^{2 n+2}$ there is a natural extension $H^{\varepsilon}(q, p)$ of $G^{\varepsilon}(q, p)$ to $\left(N, \omega_{2 n+2} \mid N\right)$ given by

$$
H^{\varepsilon}(q, p)=H_{0}(q, p)+\varepsilon H_{1}(q, p, \varepsilon)
$$

where $H_{0}$ is given by (5), and $N$ is given by (6). The system $\left(G^{\varepsilon}, T^{+} S^{n}, \omega_{2 n+2} \mid T^{+} S^{n}\right)$ is now obtained by constraining the system $\left(H^{2}, N, \omega_{2 n+2} \mid N\right)$ to $T^{+} S^{n}$. By Lemma's 10,11 , and 12 we may apply the constrained normalization algorithm of $\S 3$.

When we are in case (ii) $\hat{G}^{\varepsilon}$ is singular at the collision set $B$ given in $\S 4$. Because normalization involves averaging over the orbits of $X_{\bar{G}_{0}}$, we have to omit all the collision orbits of $X_{\hat{G}_{0}}$, i.e. the orbits passing through $B$. Therefore we consider $\left(\hat{G}^{\varepsilon}, \mathbf{T}^{+} \mathbf{S}^{n}, \omega_{2 n+2} \mid \mathbf{T}^{+} \mathbf{S}^{n}\right)$ (notation as in $\S 4$ ). This system is obtained by constraining to $\mathrm{T}^{+} \mathrm{S}^{n}$ the system $\left(H^{\varepsilon}, V, \omega_{2 n+2} \mid V\right)$, where $H^{\varepsilon}$ is given in (24) and $V=N-C_{2 n}$. Again we may apply the constrained normalization algorithm to $H^{\varepsilon}$.

Now suppose that we have obtained a normal form $\mathscr{H}^{\varepsilon}$ for $H^{\varepsilon}$ defined on $W$, where $W$ is either $N$ or $V$, using the constrained normalization algorithm. Then $\mathscr{H}^{\varepsilon}=H_{0}+\varepsilon \mathscr{H}_{1}$. If $\varphi$ is the normalizing symplectic transformation then $\mathscr{H}_{1}=H_{1} \circ \varphi$. Because of the normalization algorithm, the restrictions of $\mathscr{H}_{1}$ and $H_{0}$ to $T^{+} S^{n} \cap W$ commute under the Poisson bracket on $\left(T^{+} S^{n} \cap W, \omega_{2 n+2} \mid T^{+} S^{n} \cap W\right)$. Because $m$ is a symplectic diffeomorphism we obtain a normal form $\hat{\mathscr{K}}^{\varepsilon}=\mathscr{H}^{\varepsilon} \circ m^{-1}$ for $\hat{K}^{\varepsilon}$. More precisely $\hat{\mathscr{K}}^{\varepsilon}=\hat{K}_{0}+\varepsilon \mathscr{K}_{1}$ where $\mathscr{K}_{1}=K_{1} \circ m \circ \varphi \circ m^{-1}$. Notice that $\hat{\mathscr{K}}^{\varepsilon}$ is defined on $m\left(T^{+} S_{n p}^{n} \cap W\right)$. Going backwards through the pre-regularization process now gives a normal form for $K^{\varepsilon}$.

We will illustrate the constrained normalization algorithm with two examples: (a) the lunar problem which belongs to case (i); (b) the main problem of artificial satellite theory which belongs to case (ii). 


\section{Normalization of the lunar problem}

The name lunar problem stands for the three dimensional restricted three body problem (sun, earth, moon) when the value of the Jacobi constant is large. The primaries (sun, earth) have masses $1-v$ and $v$, and the massless body (the moon) is assumed to be confined to move in the Hill's region of the body with mass $v$ (the earth).

Following Kummer [4] this system can be formulated as a perturbated Kepler system on $\left(M=\left(\mathbb{R}^{3}-\{0\}\right) \times \mathbb{R}^{3}, \omega_{6} \mid M\right)$ with Hamiltonian

$$
\tilde{K}(x, y)=\frac{1}{2}|y|^{2}-\frac{v}{|x|}-\left(x_{1} y_{2}-x_{2} y_{1}\right)-(1-v)\left(3 x_{1}^{2}-|x|^{2}\right)+O\left(|x|^{3}\right)
$$

restricted to the energy surface $\tilde{K}=-\frac{1}{2} k^{2} \varepsilon^{-2}$, where $\varepsilon \ll k$. Stretching variables according to

$$
x=v \varepsilon^{2} \xi, \quad y=\varepsilon^{-1} \eta, \quad \tilde{K}=\varepsilon^{-2} K, \quad t=\lambda t_{\text {old }},
$$

where $\lambda=v \varepsilon^{2}$, gives

$$
\begin{aligned}
K^{\lambda}(\xi, \eta)= & \frac{1}{2}|\eta|^{2}-\frac{1}{|\xi|}-\lambda\left(\xi_{1} \eta_{2}-\xi_{2} \eta_{1}\right) \\
& -\frac{1}{2}(1-v) \lambda^{2}\left(3 \xi_{1}^{2}-|\xi|^{2}\right)+O\left(v^{-1} \lambda^{4}\right)
\end{aligned}
$$

on $K^{\lambda}=-\frac{1}{2} k^{2}$.

Going through the pre-regularization process we obtain

$$
\begin{aligned}
\hat{K}^{\lambda}(\xi, \eta)= & \frac{1}{2 k}|\xi|\left(|\eta|^{2}+k^{2}\right)-\lambda|\xi|\left(\xi_{1} \eta_{2}-\xi_{2} \eta_{1}\right) \\
& -\frac{1}{2}(1-v) \lambda^{2}|\xi|\left(3 \xi_{1}^{2}-|\xi|^{2}\right)+O\left(v^{-1} \lambda^{4}\right) .
\end{aligned}
$$

Applying the map $m$ given by (11) gives

$$
\begin{aligned}
H^{\lambda}(q, p)=|p|-\lambda & \left(\frac{1}{k}|p|\left(1-q_{4}\right)\left(q_{1} p_{2}-q_{1} p_{2}\right)\right) \\
+ & \lambda^{2}\left(\frac{3(1-v)}{2}|p|\left(q_{1} p_{2}-q_{2} p_{1}\right)+\frac{1}{2} \frac{(1-v)}{k^{2}}+\frac{3(1-v)}{2} \frac{k^{2}}{k^{2}}|p| p_{1}\right. \\
& \quad-\frac{3(1-v)}{2}|p|^{3} q_{4}-\frac{3(1-v)}{2}|p|\left(q_{1} p_{4}-q_{4} p_{1}\right) q_{4} \\
& \left.+\frac{3(1-v)}{2}|p|^{3} q_{4}^{2}-\frac{3(1-v)}{2}|p| q_{4} p_{1}-\frac{1}{2} \frac{(1-v)}{k^{3}}|p|^{3} q_{4}^{3}\right) \\
+ & O\left(v^{-1} \lambda^{4}\right) .
\end{aligned}
$$


We consider this as a formal power series in $\lambda$, writing

$$
H^{\lambda}(q, p)=H_{0}(q, p)+\lambda H_{1}(q, p)+\lambda^{2} H_{2}(q, p)+O\left(v^{-1} \lambda^{4}\right),
$$

replacing $|p|$ by $H_{0}(q, p)=\left(|q|^{2}|p|^{2}-\langle q, p\rangle^{2}\right)^{1 / 2}$. Notice that $H^{\lambda}$ is smooth on $N=\mathbb{R}^{8}-C_{8}$ (see (6)). The original system corresponds to the system $\left(H^{2}, N, \omega_{8} \mid N\right)$ constrained to $T^{+} S^{3}$.

We start our normalization process by computing the average $\bar{H}_{1}$ of $H_{1}$. Because $|p|\left(q_{1} p_{2}-q_{2} p_{1}\right)$ is an integral of $X_{H_{0}}$ we only have to compute the average $\bar{q}_{4}$ of $q_{4}$. According to formula (2),

$$
\bar{q}_{4}=\frac{1}{\pi} \int_{0}^{\pi}\left(-\frac{d}{h} \sin 2 t+\cos 2 t\right) q_{4}+\left(\frac{a}{h} \sin 2 t\right) p_{4} \mathrm{~d} t=0 .
$$

Consequently

$$
\vec{H}_{1}(q, p)=-\frac{1}{k}|p|\left(q_{1} p_{2}-q_{2} p_{1}\right)
$$

The generating function $R(q, p)$ of the normalizing transformation $\exp L_{\lambda R}$ (up to first order) is computed using (3). We have

$$
\begin{aligned}
R(q, p)= & \frac{1}{\pi} \int_{0}^{\pi} \frac{1}{k} t|p|\left(q_{1} p_{2}-q_{2} p_{1}\right)\left[\left(-\frac{d}{h} \sin 2 t+\cos 2 t\right) q_{4}+\left(\frac{a}{h} \sin 2 t\right) p_{4}\right] \mathrm{d} t \\
= & \frac{1}{k}|p|\left(q_{1} p_{2}-q_{2} p_{1}\right) \frac{1}{2 \pi} \int_{0}^{2 \pi} \frac{1}{2}\left(-\frac{d}{h} u \sin u+\frac{1}{2} u \cos u\right) q_{4} \\
& +\frac{1}{2}\left(\frac{a}{h} u \sin u\right) p_{4} \mathrm{~d} u \\
= & \frac{1}{k}|p|\left(q_{1} p_{2}-q_{2} p_{1}\right) \frac{1}{2 h}\left(\mathrm{~d} q_{4}-a p_{4}\right) \\
= & \frac{1}{2 k}|p|\left(q_{1} p_{2}-q_{2} p_{1}\right) \frac{\langle q, p\rangle q_{4}-|q|^{2} p_{4}}{\left(|q|^{2}|p|^{2}-\langle q, p\rangle^{2}\right)^{1 / 2}} \\
= & -\frac{1}{2 k} \frac{|p|\left(q_{1} p_{2}-q_{2} p_{1}\right)}{H_{0}(q, p)} \sum_{i=1}^{3}\left(q_{i} p_{4}-q_{4} p_{i}\right) q_{i}
\end{aligned}
$$

Let $F_{1}(q, p)=|q|^{2}-1$ and $F_{2}(q, p)=\langle q, p\rangle$. Then we find that

$$
\begin{aligned}
\left\{F_{1}, R\right\} & =-\frac{1}{2 k}\left[\frac{\left(q_{1} p_{2}-q_{2} p_{1}\right)}{H_{0}(q, p)} \sum_{i=1}^{3}\left(q_{i} p_{4}-q_{4} p_{i}\right)\right]\left\{|q|^{2},|p|\right\} \\
& =2 \frac{\langle q, p\rangle}{|p|^{2}} R(q, p),
\end{aligned}
$$




$$
\begin{aligned}
\left\{F_{2}, R\right\}= & -\frac{1}{2 k}\left[\frac{\left(q_{1} p_{2}-q_{2} p_{1}\right)}{H_{0}(q, p)} \sum_{i=1}^{3}\left(q_{i} p_{4}-q_{4} p_{i}\right)\right]\{\langle q, p\rangle,|p|\} \\
& -\frac{1}{2 k} \frac{\left(q_{1} p_{2}-q_{2} p_{1}\right)|p|}{H_{0}(q, p)} \sum_{i=1}^{3}\left[\left(q_{i} p_{4}-q_{4} p_{i}\right)\left\{\langle q, p\rangle, q_{i}\right\}\right] \\
= & R(q, p)-R(q, p)=0 .
\end{aligned}
$$

Since $\left\{F_{1}, R\right\}\left|T^{+} S^{3}=\left\{F_{2}, R\right\}\right| T^{+} S^{3}=0$ the normalizing transformation $\exp L_{\lambda R}$ leaves $T^{+} S^{3}$ invariant. Thus we need not compute $\mathbf{R}$.

After the first order normalization the new second order term in the Hamiltonian is

$$
\begin{aligned}
\tilde{H}_{2} & =H_{2}(q, p)+\left\{H_{1}, R\right\}+\frac{1}{2}\left\{\left\{H_{0}, R\right\}, R\right\} \\
& =H_{2}(q, p)+\left\{\bar{H}_{1}, R\right\}+\frac{1}{2}\left\{\hat{H}_{1}, R\right\},
\end{aligned}
$$

where $\hat{H}_{1}=H_{1}-\bar{H}_{1}$. To compute the average $\overline{\tilde{H}_{2}}$ of $\widetilde{H}_{2}$, we compute $\bar{H}_{2}$, $\overline{\left\{\bar{H}_{1}, R\right\}}$, and $\overline{\left\{\hat{H}_{1}, R\right\}}$. We start with $H_{2}$ which is given in (28). The computation of $\bar{H}_{2}$ comes down to finding the average of $p_{1}, q_{4}, q_{4} p_{1}, q_{4}^{2}$, and $q_{4}^{3}$. As in the computation of $\bar{H}_{1}$, one has $\bar{p}_{1}=\bar{q}_{4}=\overline{q_{4}^{3}}=0$. It remains to compute $\overline{q_{4}^{2}}$, and $\overline{q_{4} p_{1}}$. To simplify the somewhat long formulas recall that

$$
S_{i j}=q_{i} p_{j}-q_{j} p_{i} .
$$

Furthermore let

$$
\begin{aligned}
Q_{j} & =\frac{\langle q, p\rangle q_{j}-|q|^{2} p_{j}}{H_{0}(q, p)}, \\
P_{j} & =\frac{\langle q, p\rangle p_{j}-|p|^{2} q_{j}}{H_{0}(q, p)},
\end{aligned}
$$

and

$$
\begin{aligned}
& A=|q|=a^{1 / 2}, \\
& B=|p|=b^{1 / 2} .
\end{aligned}
$$

In addition write

$$
D=\langle q, p\rangle=d .
$$

Using (2) and (16) we have

$$
\begin{aligned}
& \overline{q_{i} q_{j}}= \frac{1}{\pi} \int_{0}^{\pi}\left[\left(-\frac{d}{h} \sin 2 t+\cos 2 t\right) q_{i}+\left(\frac{a}{h} \sin 2 t\right) p_{i}\right] \\
& \cdot\left[\left(-\frac{d}{h} \sin 2 t+\cos 2 t\right) q_{j}+\left(\frac{a}{h} \sin 2 t\right) p_{j}\right] \mathrm{d} t \\
&=\frac{1}{2} Q_{i} Q_{j}+\frac{1}{2} q_{i} q_{j} .
\end{aligned}
$$


Similarly

$$
\overline{q_{i} p_{j}}=-\frac{1}{2} Q_{i} P_{j}+\frac{1}{2} q_{i} p_{j}
$$

From (35) and (36) we obtain $\overline{q_{4}^{2}}$ and $\overline{q_{4} p_{1}}$. Consequently $\bar{H}_{2}$ is given by

$$
\begin{aligned}
\bar{H}_{2}(q, p)= & \frac{3}{2} \frac{(1-v)}{k^{2}} B S_{14}+\frac{1}{2} \frac{(1-v)}{k^{2}} B^{3}+\frac{3(1-v)}{4} \frac{k^{3}}{3} Q_{4}^{2}+\frac{3(1-v)}{4} \frac{(1-v)}{k^{3}} B^{2} \\
& +\frac{3}{4} \frac{(1-v)}{k^{3}} B Q_{4} P_{1}-\frac{3(1-v)}{4} \frac{(1-2)}{k^{3}} B q_{4} p_{1}
\end{aligned}
$$

The next term to be computed is $\left\{\bar{H}_{1}, R\right\}$. We find that

$$
\begin{aligned}
\left\{\bar{H}_{1}, R\right\} & =\frac{1}{4 k^{2}} \frac{\left(q_{1} p_{2}-q_{2} p_{1}\right)^{2}|p|^{2}}{H_{0}(q, p)} \sum_{i=1}^{3}\left(q_{i} p_{4}-q_{4} p_{i}\right)\left\{|p|, q_{i}\right\} \\
& =-\frac{1}{4 k^{2}} S_{12}^{2} B P_{4} .
\end{aligned}
$$

Since $P_{4}=\sum_{i=1}^{3} S_{i 4} p_{i}$ and $\bar{p}_{i}=0, \bar{P}_{4}=\sum_{i=1}^{3} S_{i 4} \bar{p}_{i}=0$. Thus

$$
\overline{\left\{\bar{H}_{1}, R\right\}}=0 \text {. }
$$

Finally we have to compute $\frac{1}{2}\left\{\hat{H}_{1}, R\right\}$. Since $\hat{H}_{1}=-\bar{H}_{1} q_{4}$ we obtain

$$
\begin{aligned}
\frac{1}{2}\left\{\hat{H}_{1}, R\right\}= & -\frac{1}{2}\left\{\bar{H}_{1}, R\right\} q_{4}-\frac{1}{2}\left\{q_{4}, R\right\} \bar{H}_{1} \\
= & \frac{1}{8 k^{2}} S_{12}^{2} B P_{4} q_{4}-\frac{1}{4 k^{2}} S_{12}^{2} Q_{4} p_{4} \\
& -\frac{1}{4 k^{2}} \frac{1}{H_{0}} S_{12}^{2} B^{2} Q_{4}^{2}+\frac{1}{4 k^{2}} \frac{1}{H_{0}} S_{12}^{2} B^{2} \sum_{i=1}^{3} q_{i}^{2} .
\end{aligned}
$$

To compute $\frac{1}{2} \overline{\left\{\hat{H}_{1}, R\right\}}$ we have to determine $\overline{p_{4}^{2}}$. As in the calculation of (35) and (36), we obtain

$$
\overline{p_{4}^{2}}=\frac{1}{2} P_{4}^{2}+\frac{1}{2} p_{4}^{2}
$$


A calculation gives

$$
\begin{aligned}
\frac{1}{2} \overline{\left\{\hat{H}_{1}, R\right\}}= & \frac{1}{8 k^{2}} \frac{1}{H_{0}} B D S_{12}^{2}\left(-\frac{1}{2} Q_{4} P_{4}+\frac{1}{2} q_{4} p_{4}\right) \\
& -\frac{1}{8 k^{2}} \frac{1}{H_{0}} B^{3} S_{12}^{2}\left(\frac{1}{2} Q_{4}^{2}+\frac{1}{2} q_{4}^{2}\right) \\
& +\frac{1}{4 k^{2}} \frac{1}{H_{0}} A^{2} S_{12}^{2}\left(\frac{1}{2} P_{4}^{2}+\frac{1}{2} p_{4}^{2}\right) \\
& -\frac{1}{4 k^{2}} \frac{1}{H_{0}} D S_{12}^{2}\left(-\frac{1}{2} Q_{4} P_{4}+\frac{1}{2} q_{4} p_{4}\right) \\
& -\frac{1}{4 k^{2}} \frac{1}{H_{0}^{3}} A^{4} B^{2} S_{12}^{2}\left(\frac{1}{2} P_{4}^{2}+\frac{1}{2} p_{4}^{2}\right) \\
& +\frac{1}{2 k^{2}} \frac{1}{H_{0}^{3}} A^{2} B^{2} D S_{12}^{2}\left(-\frac{1}{2} Q_{4} P_{4}+\frac{1}{2} q_{4} p_{4}\right) \\
& -\frac{1}{4 k^{2}} \frac{1}{H_{0}^{3}} B^{2} D^{2} S_{12}^{2}\left(\frac{1}{2} Q_{4}^{2}+\frac{1}{2} q_{4}^{2}\right) \\
& -\frac{1}{4 k^{2}} \frac{1}{H_{0}} B^{2} S_{12}^{2}\left(\frac{1}{2} Q_{4}^{2}+\frac{1}{2} q_{4}^{2}\right)+\frac{1}{4 k^{2}} \frac{1}{H_{0}} A^{2} B^{2} S_{12}^{2} .
\end{aligned}
$$

Taking the average of (33) yields

$$
\overline{\tilde{H}_{2}}(q, p)=\bar{H}_{2}(q, p)+\frac{1}{2} \overline{\left\{\hat{H}_{1}, R\right\}}
$$

where $\bar{H}_{2}$ and $\overline{\left\{\hat{H}_{1}, R\right\}}$ are given by (37) and (40). Notice that on $T^{+} S^{3}$ we have the following equalities

$$
Q_{j}\left|T^{+} S^{3}=-\frac{p_{j}}{|p|}, \quad P_{j}\right| T^{+} S^{3}=-|p| q_{j} .
$$

Thus

$$
\begin{aligned}
\widetilde{\tilde{H}_{2}}(q, p) \mid T^{+} S^{3}= & \left(\frac{3}{2} \frac{(1-v)}{k^{2}}+\frac{3}{4} \frac{(1-v)}{k^{3}}\right)|p|\left(q_{1} p_{4}-q_{4} p_{1}\right)+\frac{1}{2} \frac{(1-v)}{k^{2}}|p|^{3} \\
& +\frac{3(1-v)}{4}|p|\left(|p|^{2} q_{4}^{2}+p_{4}^{2}\right) \\
& -\frac{1}{16 k^{2}}\left(q_{1} p_{2}-q_{2} p_{1}\right)^{2}\left(|p|^{2} q_{4}^{2}+p_{4}^{2}\right) \\
& +\frac{1}{4 k^{2}}\left(q_{1} p_{2}-q_{2} p_{1}\right)^{2}|p| \\
& -\frac{1}{8 k^{2}} \frac{1}{|p|}\left(q_{1} p_{2}-q_{2} p_{1}\right)^{2}\left(|p|^{2} q_{4}^{2}+p_{4}^{2}\right) .
\end{aligned}
$$


Using the fact that on $T^{+} S^{3}$ we have the relations and

$$
|p|^{2} \mid T^{+} S^{3}=\sum_{1 \leqq i<j \leqq 4}\left(q_{i} p_{j}-q_{j} p_{i}\right)^{2} \neq 0,
$$

$$
\left(|p|^{2} q_{4}^{2}+p_{4}^{2}\right) \mid T^{+} S^{3}=\sum_{k=1}^{3}\left(q_{k} p_{4}-q_{4} p_{k}\right)^{2}, \quad \text { (see [1], page 137) }
$$

we find that the normal form $\bar{H}_{1}+{\widetilde{\tilde{H}_{2}}}_{2}$ on $T^{+} S^{3}$ is equal to a smooth function in the quadratic functions $S_{i j}$ defined in (17). On $T^{+} S^{3}$ consider the $H_{0}$ level set corresponding to $|p|=L=\frac{1}{k}$. Applying the inverse of Moser's regularization map and the inverse of the pre-regularization process gives the normal form for the lunar problem to second order on the $K_{0}=-\frac{1}{2} k^{2}$ level set.

\section{The main problem of artificial satellite theory}

In this section we discuss the main problem of artificial satellite theory. This is the problem in which a point mass moves subject to the gravitational forces of an oblate sphere. In the perturbation term of the potential due to the oblateness only the dominant term is taken into account.

According to Deprit $([3]$, page 114,130$)$ the Hamiltonian of the main problem of artificial satellite theory in Whittaker coordinates $(r, \theta, v, R, \Theta, N)$ is

$$
M=\frac{1}{2}\left(R^{2}+\frac{\Theta^{2}}{r^{2}}\right)-n_{e} N-\frac{\mu}{r}\left[1-\varepsilon\left(\frac{\alpha}{r}\right)^{2}\left(1-\frac{3}{4} s^{2} \cos 2 \theta\right)\right] .
$$

Assuming $\Theta \neq 0$ we may eliminate the parallax. In mixed Whittaker and Delaunay variables, the latter given by $(l, g, h, L, G, H)$, (44) becomes

$$
M=M_{0,0}+\frac{\Theta^{2}}{r^{2}} \sum_{n \geqq 1} \frac{\varepsilon^{n}}{n !}\left(\frac{\alpha}{p}\right)^{2 n} \sum_{0 \leqq j \leqq \frac{1}{2} n} e^{2 j} \sum_{0 \leqq k \leqq j} M_{n, j, k}^{*}\left(s^{2}\right) s^{2 k} \cos 2 k g,
$$

where $M_{n, j, k}^{*}\left(s^{2}\right)$ are the inclination polynomials ([3], page 137, 138), and

$$
M_{0,0}=\frac{1}{2}\left(R^{2}+\frac{\Theta^{2}}{r^{2}}\right)-\frac{\mu}{r}-n_{e} N=M_{0}-n_{e} N
$$

is the Kepler Hamiltonian $M_{0}$ with added constant $-n_{e} N$. After using the identities $\Theta=G$ and $p=\frac{G^{2}}{\mu}$ and rearranging the terms, the Hamiltonian (45)
takes the form

$$
M=M_{0}+\frac{G^{2}}{r^{2}} \sum_{n \geqq 0} \frac{\varepsilon^{n}}{n !}\left(\frac{\alpha \mu}{G}\right)^{2 n} \sum_{0 \leqq j \leqq \frac{1}{2} n} P_{n, j}\left(e^{2}, s^{2}\right)(e s \sin g)^{2 j},
$$

where the eccentricity-inclination polynomials $P_{n, j}$ are given in Table I for $n \leqq 4$. 
Table I

Eccentricity-inclination polynomials.

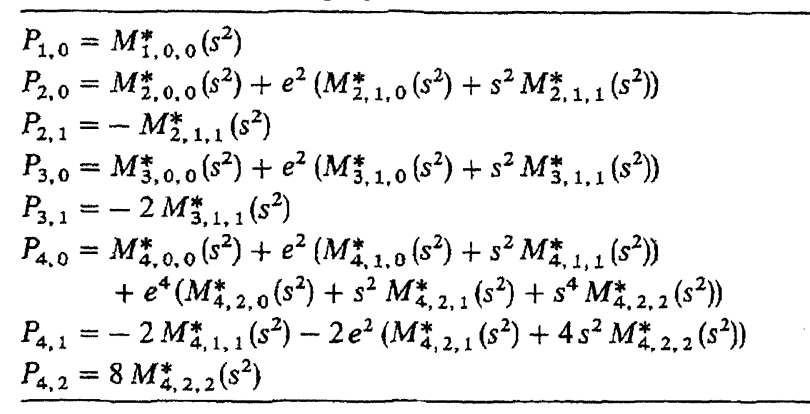

Let $J=\left(J_{1}, J_{2}, J_{3}\right)$ and $A=\left(A_{1}, A_{2}, A_{3}\right)$ be the angular momentum and Laplace vectors for the Kepler problem when $n=3$. We have the following relations

$$
\begin{gathered}
M_{0}=-\frac{\mu^{2}}{2 L^{2}}, \quad G^{2}=J_{1}^{2}+J_{2}^{2}+J_{3}^{2}, \quad G^{2} s^{2}=J_{1}^{2}+J_{2}^{2}, \\
H=J_{3}, \quad \text { es } \operatorname{sing}=A_{3}, \quad L^{2} e^{2}=L^{2}-\left(J_{1}^{2}+J_{2}^{2}+J_{3}^{2}\right) .
\end{gathered}
$$

Using (47) we may express the Delaunay variables in (46) in terms of $L$, and the components of $J$ and $A$. Thus the Hamiltonian of the main problem after elimination of the parallax has the form

$$
K^{\varepsilon}(\xi, \eta)=K_{0}(\xi, \eta)+\varepsilon \frac{K_{1}(\xi, \eta, \varepsilon)}{|\xi|^{2}},
$$

where $K_{0}(\xi, \eta)$ is the Kepler Hamiltonian (4) and $\left\{K_{0}, K_{1}\right\}=0$, because $K_{1}$ is a smooth formal power series in $J_{i}, A_{i}, i=1,2,3$, and $L$ which are integrals of $X_{K_{0}}$.

Recall that for $\varepsilon=0$ we consider only those orbits whose total energy is negative. Because after regularization the perturbation term in (48) can not be extended to a smooth function on $T^{+} S^{3}$ we must consider those orbits of the unperturbed Kepler system with nonzero angular momentum. After preregularization (48) becomes

$$
\hat{K}^{\varepsilon}(\xi, \eta)=\frac{1}{2 k}|\xi|\left(|\eta|^{2}+k^{2}\right)+\varepsilon \frac{k K_{1}(\xi, \eta, \varepsilon)}{|\xi|} .
$$

Applying Moser's diffeomorphism $m$ to $\hat{K}^{\varepsilon}$ yields a Hamiltonian system $\left(\hat{G}^{\varepsilon}, \mathbf{T}^{+} \mathbf{S}^{3}, \omega_{2 n+2} \mid \mathbf{T}^{+} \mathbf{S}^{\mathbf{3}}\right)$. Here

$$
\hat{G}^{\varepsilon}(q, p)=G_{0}(q, p)+\varepsilon \hat{F}(q, p) \hat{G}_{1}(q, p, \varepsilon),
$$

where $G_{0}(q, p)=|p|, \hat{F}(q, p)=\left(1-q^{4}\right)^{-1}$, and $\hat{G}_{1}(q, p, \varepsilon)=\frac{k}{|p|} K_{1} \circ m(q, p, \varepsilon)$. 
Consider the polynomials $S_{i j}=q_{i} p_{j}-q_{j} p_{i}$ on $\mathbb{R}^{8}$. On $G_{0}^{-1}(L) \subseteq \mathbb{R}^{8}$, where $L=\frac{\mu}{k}$, we have

$$
\begin{aligned}
& J_{1} \circ m=S_{23}, \quad J_{2} \circ m=S_{13}, \quad J_{3} \circ m=S_{12} . \\
& A_{1} \circ m=-\frac{1}{L} S_{14}, \quad A_{2} \circ m=-\frac{1}{L} S_{24}, \quad A_{3} \circ m=-\frac{1}{L} S_{34} .
\end{aligned}
$$

We consider these as smooth functions on $\mathrm{T}^{+} \mathrm{S}^{3}$ instead of $G_{0}^{-1}(L)$. Because $K_{1}$ is a smooth function of $L, J_{i}, A_{i}, i=1,2,3$, it follows that $\hat{G}_{1}$ is a smooth function of $S_{i j} \mid \mathbf{T}^{+} \mathbf{S}^{3}, 1 \leqq i<j \leqq 4$. Because Moser's map $m$ is a symplectic diffeomorphism from $\left(\mathbf{T}^{+} \mathbf{S}^{3}, \omega_{8} \mid \mathbf{T}^{+} \mathbf{S}^{3}\right)$ onto $\left(\left(\mathbb{R}^{3}-\{0\}\right) \times \mathbb{R}^{3}\right)$ $-m\left(C_{6} \cap T^{+} S^{3}\right)$ with symplectic form equal to the restriction of $\omega_{6}, \hat{G}_{1}$ is a formal power series integral of $X_{\hat{G}_{0}} \mid \mathbf{T}^{+} \mathbf{S}^{3}$.

We may now apply the constrained normalization process on the Hamiltonian system $\left(H^{\varepsilon}, V, \omega_{8} \mid V\right)$, where $V=\mathbb{R}^{8}-C_{8} \cap C_{6}$, and $H^{\varepsilon}$ is given by

$$
H^{\varepsilon}(q, p)=H_{0}(q, p)+\varepsilon F(q) H_{1}(q, p, \varepsilon),
$$

with $H_{0}(q, p)=\left(|q|^{2}|p|^{2}-\langle q, p\rangle^{2}\right)^{1 / 2}, F(q)=\left(|q|-q_{4}\right)^{-1}$, and $H_{1}$ the smooth extension of $\hat{G}_{1}$ to $V$ defined by $H_{1}=\hat{G}_{1}\left(S_{i j} \mid V, \varepsilon\right)$. Note that $H_{1}$ is a smooth formal power series integral of $X_{H_{0}}$ and that $H^{\varepsilon}$ is a smooth extension of $\hat{G}^{\varepsilon}$.

To compute the constrained normal form for $H^{\varepsilon}$ we have to compute $\overline{F \cdot H_{1}}=\bar{F} \cdot H_{1}$. Using (2) and (16) we obtain

$$
\begin{aligned}
\bar{F} & =\frac{1}{\pi} \int_{0}^{\pi}\left(a^{1 / 2}-\left[\left(-\frac{d}{h} \sin 2 t+\cos 2 t\right) q_{4}-\left(\frac{a}{h} \sin 2 t\right) p_{4}\right]\right)^{-1} \mathrm{~d} t \\
& =\frac{1}{2 \pi a^{1 / 2}} \int_{0}^{2 \pi}\left(1+\left(\frac{d}{a^{1 / 2} h} q_{4}-\frac{a^{1 / 2}}{h} p_{4}\right) \sin v-\frac{q_{4}}{a^{1 / 2}} \cos v\right)^{-1} \mathrm{~d} v .
\end{aligned}
$$

If we let

$$
\tilde{e}=\left[\left(\frac{d}{a^{1 / 2} h} q_{4}-\frac{a^{1 / 2}}{h} p_{4}\right)^{2}+\frac{1}{a} q_{4}^{2}\right]^{1 / 2},
$$

and choose $\chi$ so that

$$
\tilde{e} \cos \chi=\frac{q_{4}}{a^{1 / 2}}, \quad \tilde{e} \sin \chi=\frac{d}{a^{1 / 2} h} q_{4}-\frac{a^{1 / 2}}{h} p_{4},
$$

then (53) becomes

$$
a^{1 / 2} \bar{F}=\frac{1}{2 \pi} \int_{0}^{2 \pi} \frac{1}{1-\tilde{e} \cos (v+\chi)} \mathrm{d} v .
$$


Before we compute (56) we digress to show that $\tilde{e}$ is a smooth extension of $e \circ m$, where $e$ is the eccentricity defined in (47). On $Q=G_{0}^{-1}(L)$ $-\left(C_{6} \cap T^{+} S^{3}\right) \subseteq \mathrm{T}^{+} \mathrm{S}^{3}$ the integrals $|q|^{2}=a,|p|^{2}=b,\langle q, p\rangle=d, H_{0}=h$ of $X_{H_{0}}$ on $V$ take the values $1, L^{2}, 0$, and $L$ respectively. Therefore

$$
\begin{aligned}
\tilde{e}^{2} \mid Q & =\left(\frac{a}{h^{2}} p_{4}^{2}+\frac{1}{a} q_{2}^{2}\right)\left|Q=\frac{1}{L^{2}}\left(|p|^{2} q_{4}^{2}+p_{4}^{2}\right)\right| Q \\
& =\left(A_{1}^{2}+A_{2}^{2}+A_{3}^{2}\right) \circ m\left|Q=e^{2} \circ m\right| Q .
\end{aligned}
$$

Here we have used (43). The following argument shows that on $V$ the function $\tilde{e}$ takes values in $[0,1)$. Since

$$
\frac{q_{4}(v)}{|q(v)|}=\left(\varphi_{\frac{1}{2} v}^{H_{0}}\right) * \frac{q_{4}}{|q|}=\tilde{e} \cos (v+\chi)
$$

it follows that $\tilde{e}=1$ if and only if for some $\tilde{v} \in[0,2 \pi] q_{4}(\tilde{v})=|q(\tilde{v})|$, that is, if and only if $q_{1}(\tilde{v})=q_{2}(\tilde{v})=q_{3}(\tilde{v})=0$ and $q_{4}(\tilde{v})>0$. But then $(q(\tilde{v}), p(\tilde{v}))$ lies on $C_{6}$ and consequently it does not lie in $V$. Therefore the integrand of (56) is defined. We have

$$
\begin{aligned}
a^{1 / 2} \bar{F} & =\frac{1}{2 \pi} \int_{0}^{2 \pi} \frac{1}{1-\tilde{e} \cos (v+\chi)} \mathrm{d} v=\frac{1}{2 \pi} \int_{x}^{2 \pi+\chi} \frac{1}{1-\tilde{e} \cos u} \mathrm{~d} u \\
& =\frac{1}{2 \pi} \int_{0}^{2 \pi} \frac{1}{1-\tilde{e} \cos u} \mathrm{~d} u=\frac{1}{\sqrt{1-\tilde{e}^{2}}} .
\end{aligned}
$$

By Lemma 7 we obtain the normalizing transformation $\exp L_{\varepsilon \mathbf{R}}$ (see also Lemma 2 and Theorem 8) where

$$
R=\frac{1}{\pi} \int_{0}^{\pi} t\left(\varphi_{t}^{H_{0}}\right)^{*}\left(F \cdot H_{1}-\bar{F} \cdot H_{1}\right) \mathrm{d} t=H_{1} \cdot \tilde{F}
$$

and

$$
\tilde{F}=\frac{1}{\pi} \int_{0}^{\pi} t\left(\varphi_{t}^{H_{0}}\right)^{*}(F-\bar{F}) \mathrm{d} t .
$$

It remains to calculate $\widetilde{F}$. Using (58) and (59) we find that

$$
\begin{aligned}
a^{1 / 2} \tilde{F} & =\frac{1}{2 \pi} \int_{0}^{2 \pi} t\left[\frac{1}{1-\tilde{e} \cos (t+\chi)}-\frac{1}{\sqrt{1-\tilde{e}^{2}}}\right] \mathrm{d} t \\
& =\frac{1}{2 \pi} \int_{0}^{2 \pi} \frac{t+\chi}{1-\tilde{e} \cos (t+\chi)} \mathrm{d} t-\frac{\chi}{2 \pi} \int_{0}^{2 \pi} \frac{1}{1-\tilde{e} \cos (t+\chi)} \mathrm{d} t-\frac{\pi}{\sqrt{1-\tilde{e}^{2}}} \\
& =\frac{1}{2 \pi} \int_{\chi}^{2 \pi+\chi} \frac{u}{1-\tilde{e} \cos u} \mathrm{~d} u-\frac{\chi+\pi}{\sqrt{1-\tilde{e}^{2}}}
\end{aligned}
$$


but

$$
\begin{aligned}
\frac{1}{2 \pi} \int_{\chi}^{2 \pi+\chi} \frac{u}{1-\tilde{e} \cos u} \mathrm{~d} u= & \frac{1}{2 \pi}\left(\int_{x}^{0}+\int_{0}^{2 \pi}+\int_{2 \pi}^{2 \pi+\chi}\right) \frac{u}{1-\tilde{e} \cos u} \mathrm{~d} u \\
= & \frac{1}{2 \pi}\left(\int_{\chi}^{0}+\int_{0}^{2 \pi}\right) \frac{u}{1-\tilde{e} \cos u} \mathrm{~d} u \\
& +\frac{1}{2 \pi} \int_{0}^{\chi} \frac{v+2 \pi}{1-\tilde{e} \cos (v+2 \pi)} \mathrm{d} v \\
= & \frac{1}{2 \pi} \int_{0}^{2 \pi} \frac{u}{1-\tilde{e} \cos u} \mathrm{~d} u+\int_{0}^{\chi} \frac{1}{1-\tilde{e} \cos u} \mathrm{~d} u \\
= & \frac{\pi}{\sqrt{1-\tilde{e}^{2}}}+\frac{2}{\sqrt{1-\tilde{e}^{2}}} \tan ^{-1}\left[\left(\frac{1+\tilde{e}}{1-\tilde{e}}\right)^{1 / 2} \tan \frac{\chi}{2}\right]
\end{aligned}
$$

Therefore on $V$

$$
a^{1 / 2} \tilde{F}=\frac{2}{\sqrt{1-\tilde{e}^{2}}} \tan ^{-1}\left[\left(\frac{1+\tilde{e}}{1-\tilde{e}}\right)^{1 / 2} \tan \frac{\chi}{2}\right]-\frac{\chi}{\sqrt{1-\tilde{e}^{2}}} .
$$

This completes the computations.

Note that because $\tilde{e}^{2}=\frac{1}{L^{2}}\left(S_{14}^{2}+S_{24}^{2}+S_{34}^{2}\right)$ on $\mathrm{T}^{+} \mathbf{S}^{3}$ we find that the restriction of our normal form to $\mathbf{T}^{+} \mathbf{S}^{3}$ is a smooth function in the $S_{i j} \mid \mathbf{T}^{+} \mathbf{S}^{3}$.

\section{Acknowledgement}

The first ideas about constrained normalization developed during conversation between the two authors when they were both at the University of Utrecht. The first author continued the investigations while he was visiting the Forschungsinstitut für Mathematik of the E.T.H. Zürich, made possible by a stipendium of this institute. He would like to thank Prof. Moser for his invitation and for his valuable comments on an earlier draft of the paper. The second author worked on this paper while he was visiting the University of California at Santa Cruz. He would like to thank the Mathematics Board of Studies for its hospitality.

\section{References}

[1] R. Cushman, Normal forms for Hamiltonian vector fields with periodic flow. In: Differential Geometric Methods in Mathematical Physics, ed. S. Sternberg, 125-144, D. Reidel, Dordrecht 1984.

[2] P. Deift, F. Lund and E. Trubowitz, Nonlinear wave equations and constrained harmonic motion. Comm. Math. Phys. 74, 144-188 (1980). 
[3] A. Deprit, The elimination of the parallax in satellite theory. Celest. Mech. 24, 111-153 (1981).

[4] M. Kummer, On the 3-dimensional lunar problem and other perturbations of the Kepler problem. J. Math. Anal. Appl. 93, 142-194 (1983).

[5] J. Moser, Regularization of Kepler's problem and the averaging method on a manifold. Comm. Pure Appl. Math. 23, 609-636 (1970).

[6] J. Moser, Geometry of quadrics and spectral theory. In: The Chern Symposium 1979, ed. W.-Y. Hsiang et. al., 147-188, Springer Verlag, New York 1980.

\section{Summary}

Consider a Hamiltonian system $\left(H, \mathbb{R}^{2 n}, \omega\right)$. Let $M$ be a symplectic submanifold of $\left(\mathbb{R}^{2 n}, \omega\right)$. The system $\left(H, \mathbb{R}^{2 n}, \omega\right)$ constrained to $M$ is $(H|M, M, \omega| M)$. In this paper we give an algorithm which normalizes the system on $\mathbb{R}^{2 n}$ in such a way that restricted to $M$ we have normalized the constrained system. This procedure is then applied to perturbed Kepler systems such as the lunar problem and the main problem of artificial satellite theory.

\section{Zusammenfassung}

Wir betrachten ein Hamiltonisches System $\left(H, \mathbb{R}^{2 n}, \omega\right)$. Sei $M$ ein symplectisches Submanifold von $\left(\mathbb{R}^{2 n}, \omega\right)$. Das System $\left(H, \mathbb{R}^{2 n}, \omega\right)$, auf $M$ beschränkt, ist $(H|M, M, \omega| M)$. In der vorliegenden Arbeit wird ein Algorithmus vorgeschlagen, der dieses System so auf $\mathbb{R}^{2 n}$ normalisiert, daß das auf $M$ beschränkte System auch normalisiert ist. Dieser Algorithmus wird dann auf gestörte Keplersysteme, wie z. B. das Hill-sche Mondproblem und das Hauptproblem der Theorie der künstlichen Satelliten, angewendet.

(Received: Janusary 17, 1986) 